\title{
Applying Testability Transformations to Achieve Structural Coverage of Erlang Programs
}

\author{
Qiang Guo, John Derrick, and Neil Walkinshaw \\ Department of Computer Science, \\ The University of Sheffield, \\ Regent Court, 211 Portobello, S1 4DP, UK \\ $\{$ Q.Guo, J.Derrick, N.Walkinshaw\}@dcs.shef .ac.uk
}

\begin{abstract}
This paper studies the structural testing of Erlang applications. A program transformation is proposed that represents the program under test as a binary tree. The challenge of achieving structural coverage can thus be interpreted as a tree-search procedure. We have developed a testing-technique that takes advantage of this tree-structure, which we demonstrate with respect to a small case study of an Erlang telephony system.
\end{abstract}

Keywords: Erlang, Testing, Transformation, FBT, Structural Coverage.

\section{Introduction}

Erlang [1] was, along with its Open Telecoms Platform (OTP), originally developed by Ericsson for the rapid development of network applications. However, its usage has now spread beyond that domain to a number of sectors. Erlang has been designed to provide a paradigm for the development of distributed soft real-time systems, where multiple processes can be spread across many nodes in a network.

With its OTP libraries, complex Erlang applications can be rapidly developed and deployed across a large variety of hardware platforms, and this has caused it to become increasingly popular, not only within large telecoms companies such as Ericsson, but also with a variety of SMEs in different areas. It is increasingly used to develop applications that are business-critical, for example, its use in Ericsson's AXD-301 switch that provides British Telecom's internet backbone.

The ability to rigorously test Erlang applications is vital. In recognition of this, there has recently been a concerted drive to develop more automated testing tools, which complement the rapid Erlang development cycle. Tools such as QuickCheck [2] are being eagerly adopted within the community.

So far, the main thrust of the Erlang testing effort has been directed towards functional testing. Structural code-coverage has not been considered. This is however an important problem, and is often a requirement if the system under test is to be certified for a range of safety standards (e.g. the US Federal Aviation Authority software standards [13). Achieving code coverage is a wellestablished challenge; the tester has to find a suitable set of test inputs that 
will exercise every branch for every predicate in the program. Finding a suitable (and reasonably sized) set of inputs is often problematic, and a number of techniques such as symbolic execution [7], evolutionary search algorithms [9] and testability-transformations [6] have been proposed to address it.

This paper presents a technique that leverages certain features of the Erlang language to enable complete structural test coverage. The approach is inspired by Harman et al.'s notion of testability transformations [6. We have developed a transformation that transforms an Erlang program into a binary tree. Each node in the tree corresponds to either a true or a false evaluation of a predicate in the original program. Predicates that may be hidden within functions in the original program are made explicit. Identifying a suitable test set to achieve structural coverage is thus reduced to a search over the binary tree.

To facilitate the debugging process, a simple debugging framework is presented. The framework takes advantage of the tree structure of the transformed

program. This enables the developer to control and observe the execution of a program by directly manipulating the internal variable and parameter values.

The paper is structured as follows. Section 2 provides a background to Erlang and the structural testing problem. Section 3 introduces our program transformation. Section 4 shows how tests can be generated to cover the transformed program. Section [5 contains a case study with respect to a small telecommunications system, and section 6 contains conclusions and future work.

\section{Background}

This section provides a brief introduction to Erlang.

\section{$2.1 \quad$ Erlang}

Erlang is a concurrent functional language with specific support for the development of distributed,fault-tolerant systems with soft real-time requirements [1]. It was designed from the start to support a concurrency-oriented programming paradigm and large distributed implementations that this supports.

The Open Telecom Platform (OTP) is a set of Erlang libraries for building large fault-tolerant distributed applications. Extensive libraries for common network-applications and protocols are included, and there is a collection of source code and trace-analysis tools that provide a base for debugging and profiling tasks. It also provides a set of common and reusable behaviours, which encapsulate common behavioural patterns for processes, where the library module implements the generic behaviour, and the developer is left to add those aspects of behaviour that are specific to their system.

With the OTP, Erlang applications can be rapidly developed and deployed across a large variety of hardware platforms, and this has caused it to become increasingly popular, not only within large telecoms companies such as Ericsson, but also with a variety of SMEs in different areas such as Yahoo! Delicious, and the Facebook chat system. 
However, verification and validation of Erlang systems is to-date a largely adhoc, manual process. Consequently there is an inherent danger that important functionality remains untested and undocumented. Thus along with its recent growth in popularity, there has been a concerted drive to develop more automated and systematic techniques.

\subsection{Structural Testing of Erlang Implementations}

In structural testing, the goal is to achieve some level of coverage of the source code. Branch coverage, where the aim is to cover every branch from every decision point in the source code, is one of the most popular measures. The challenge of generating a suitable test set is two-fold: (1) to identify a suitable set of test inputs that will reach every branch, and (2) to ensure that this set of tests is sufficiently small so that can be executed in a reasonable amount of time.

An Erlang program consists of a set of modules, each of which defines a number of functions. Functions that are accessible from other modules need to be explicitly declared by the export command. A function named f_name in the module module and with arity $N$ is denoted as module:f_name/N. An Erlang function $f_{i}$ is coded as a sequence of executional units $f_{i}=\left\langle c p_{i(1)}, \ldots\right.$, $\left.c p_{i(n)}\right\rangle$, each of which defines a set of statements. An example is demonstrated in Figure 1

From the structural-testing perspective, the aim is to identify a set of testinputs that will collectively ensure that every branch in client:idle is executed at least once. Along with the input parameters, it is also necessary for the tester to control the values that are returned by gen_server. In this case, we would need to ensure that the variable $P 1$ is at some point assigned to one of the four predicate conditions (e.g. \{error,invalid_mobile\}), as well as an aribitrary value that corresponds to none of them.
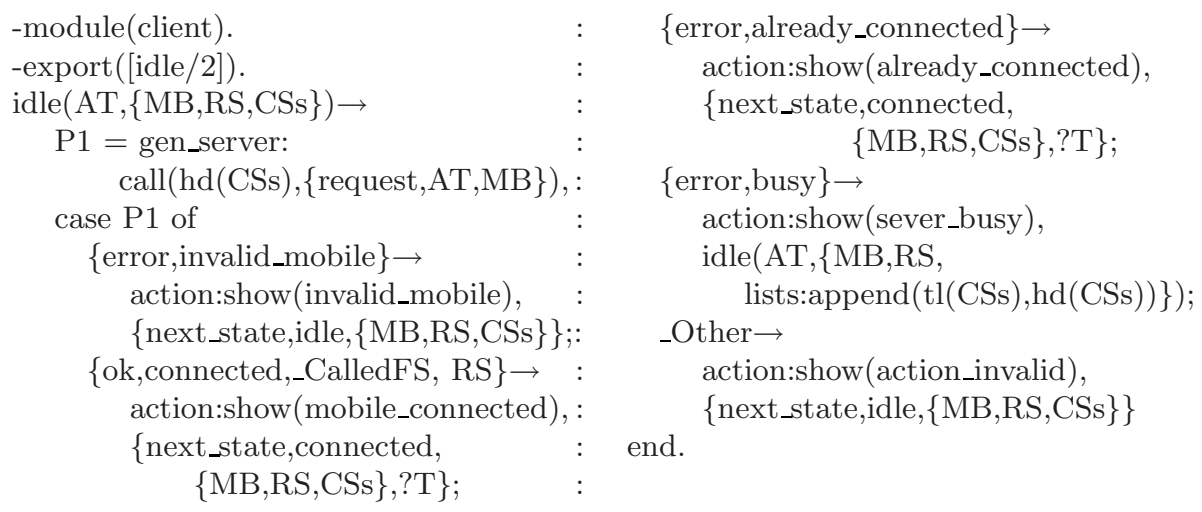

Fig. 1. An Erlang Example 
The simplest strategy is to construct a separate test set for each branch. For each unit $c p_{i(k)}$ a test set is designed and applied to $f_{i}$. The $\mathrm{I} / \mathrm{O}$ behavior observed from $\left\langle c p_{i(1)}, \ldots, c p_{i(k)}\right\rangle$ is used to evaluate $c p_{i(k)}$. When the test of $c p_{i(k)}$ is complete, the process continues to the next unit. The process continues until all units have been tested.

Such a test strategy could give rise to two disadvantages. First, this approach can be wasteful; testing $c p_{i(k)}$ requires the execution of all preceding units, $\left\{c p_{i(1)}, \ldots, c p_{i(k-1)}\right\}$, regardless of whether $c p_{i(l)}, l<l<k$, has already been tested. Secondly, identifying a suitable combination of inputs can be very challenging. The execution of $c p_{i(k)}$ is heavily dependent by the results of executing $\left\langle c p_{i(1)}, \ldots, c p_{i(k-1)}\right\rangle$ as the parameters for $c p_{i(k)}$ may be affected by previous results.

From this small example, it is possible to identify the input requirements by hand, simply from inspecting the predicate conditions. However, this approach becomes intractable when the software increases in scale and complexity. Given a conventional Erlang system with multiple modules, where certain branches can only be reached by complex combinations of conditions, an automated approach is required.

\section{Transforming an Erlang Program into Binary Format}

This paper proposes a program transformation that converts an Erlang function into a tree-structure. The transformed function retains the functional behavior, but makes the predicates that control its behavior explicit. This has two benefits that address the problem mentioned above. The tree-shaped structure makes it possible to identify an efficient test-set. Making the predicates explicit makes it possible to automatically identify the set of inputs that are required to reach every unit in a function. The rest of this section will introduce the program transformation.

\subsection{Notations}

To formalize the description, we introduce the following notations. Let $\left.c p_{i(k)}\right|_{\operatorname{cond}(k)}$ denote that the unit $c p_{i(k)} \in f_{i}$ is executed under the condition of $\operatorname{cond}(k)$. If $\operatorname{cond}(k)=$ true, $c p_{i(k)}$ is activated for execution; otherwise a dummy operation 11 is performed. Let $c p_{i(l)}<<c p_{i(m)}$ denote that $c p_{i(m)}$ is executed after $c p_{i(l)}$. The function $f_{i}$ can be expressed as $f_{i}=\left.c p_{i(1)}\right|_{\operatorname{cond}(i(1))}$ $<<\ldots<<\left.c p_{i(n)}\right|_{\operatorname{cond}(i(n))},\left.c p_{i(l)}\right|_{\operatorname{cond}(i(l))}<<\left.c p_{i(m)}\right|_{f a l s e}<<\left.c p_{i(n)}\right|_{\operatorname{cond}(i(n))} \equiv$ $\left.c p_{i(l)}\right|_{\operatorname{cond}(i(l))}<<\left.c p_{i(n)}\right|_{\operatorname{cond}(i(n))}, \forall(l<m<n)$. If $c p_{i(l)} \in f_{i}$ is an unconditional unit, $\operatorname{cond}(i(l))$ is automatically set to true; otherwise, the value of $\operatorname{cond}(i(l))$ is determined by the pattern evaluation. For example, the function idle shown in Figure 1 is expressed as:

\footnotetext{
${ }^{1}$ A dummy operation is a function that maps the inputs to the outputs without
} changing the values of the inputs. 


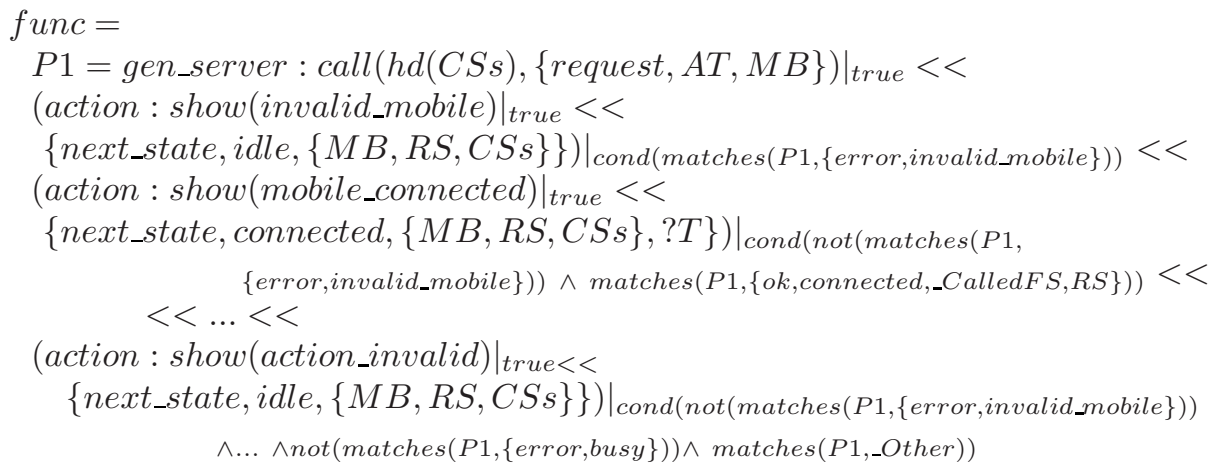

where the function matches evaluates whether $P 1$ matches $P V, P V \in\{\{$ error, invalid_mobile $\},\{$ ok, connected,_CalledFS, $R S\},\{$ error, already_connected $\},\{$ error, busy\},_Other $\}$.

The above expressions show how each unit $c p_{i(k)} \in f_{i}$ can be represented by a generic pattern, $\left.c p_{i(k)}\right|_{\operatorname{cond}(i(k))}$. If $\operatorname{cond}(i(k))$ is true, $c p_{i(k)}$ is executed; otherwise, $\left.c p_{i(k+1)}\right|_{\operatorname{cond}(i(k+1))}$ is evaluated.

\subsection{Function Transformation}

The transformation we introduce here decomposes a single large function $f_{i}$ into a set of atomic functions $f_{i(k)}^{\prime}$. Each function $f_{i}$ is transformed into a set of ordered calls to atomic functions $f_{i(1)}^{\prime}<<\ldots<<f_{i(n)}^{\prime}$ where $n$ is the number of executional units of $f_{i}$. The benefit of this is that the guards on the execution of these atomic functions are made explicit, and it becomes easier to control their execution, or even influence their execution for the purpose of debugging (this is demonstrated in the following sections).

The unit $c p_{i(k)} \in f_{i}$ is transformed into a called function defined as:

$$
\begin{aligned}
& f_{i(k)}^{\prime}(\text { true, Args }) \rightarrow c p_{i(k)} ; \\
& f_{i(k)}^{\prime}(\text { false, Args }) \rightarrow f_{i(k+1)}^{\prime}(\operatorname{cond}(i(k+1)), \text { Args }) .
\end{aligned}
$$

The function $f_{i(k)}^{\prime}$ is guarded by a switch that can only be set to true or false. If switch is true $($ cond $(i(k))=$ true $)$, the functional executions defined in $c p_{i(k)}$ are performed; otherwise, the function $f_{i(k+1)}^{\prime}$ is invoked where $\left.c p_{i(k+1)}\right|_{\operatorname{cond}(i(k+1))}$ is evaluated.

When $f_{i(k)}^{\prime}$ is invoked, if $f_{i(k)}^{\prime}$ is derived from a unconditional unit $c p_{i(k)}$, switch is automatically set to true; otherwise, the pattern match evaluation function patterns_match is applied to decide the value of switch. Definition of the function patterns_match is discussed in the subsection 3.3. According to the pattern under evaluation, the function patterns_match returns true or false. If $c p_{i k} \in f_{i}$ defines a list of variables $R V_{k}$, when $c p_{i m} \in f_{i}, m>k$, is transformed to $f_{i(m)}^{\prime}, R V_{k}$ is configured as an argument of $f_{i(m)}^{\prime}$. 


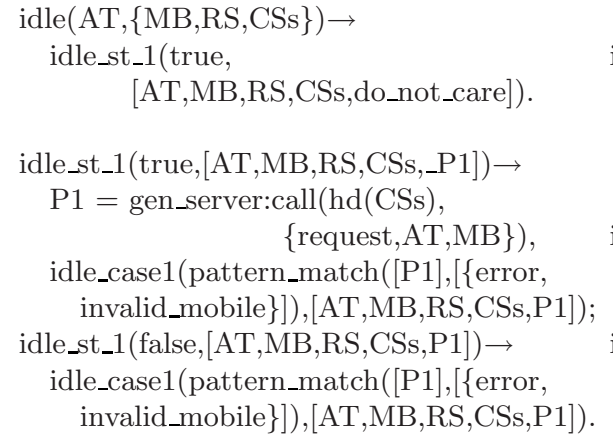

\{next_state,connected, $\{\mathrm{MB}, \mathrm{RS}, \mathrm{CSs}\}, ? \mathrm{~T}\}$;

idle_case2(false, [AT,MB,RS,CSs,P1]) $\rightarrow$

idle_case3(pattern_match $([\mathrm{P} 1],[\{$ error, already_connected $\}]$ ),

$[\mathrm{AT}, \mathrm{MB}, \mathrm{RS}, \mathrm{CSs}, \mathrm{P} 1])$.

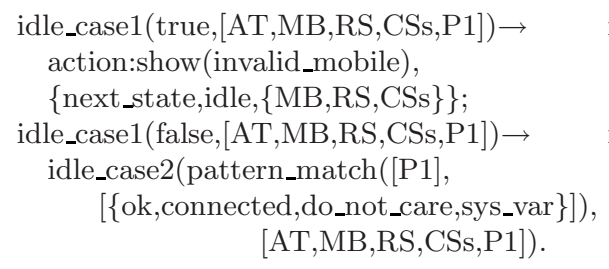

idle_case2(true,[AT,MB,RS,CSs,P1]) $\rightarrow$ action:show(mobile_connected),

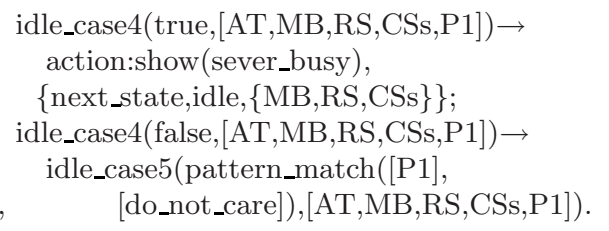

idle_case5(true,[AT,MB,RS,CSs,P1]) $\rightarrow$ action:show(action_invalid), $\{$ next_state,idle, $\{\mathrm{MB}, \mathrm{RS}, \mathrm{CSs}\}\}$

Fig. 2. Transformed Erlang program

Thus, the defined rules transform the original function $f_{i}$ into a set of calls to functions $\left\{f_{i(1)}^{\prime}, \ldots, f_{i(n)}^{\prime}\right\}$ where $n$ is the number of units defined in $f_{i}$. Each function $f_{i(k)}^{\prime}$ is coded by generic pattern with a true branch and a false branch being defined. The true branch performs the functional executions defined in $c p_{i k} \in f_{i}$, while, the false branch invokes the function $f_{i(k+1)}^{\prime}$ where cond $(i(k+1))$ is evaluated. Such a transformation is called Binary Transformation (BT). The true branch of a called function is called Functional Execution Branch (FEB) and the false branch is called Pattern Evaluation Branch (PEB). By applying the defined rules, the function idle shown in Figure 1 is transformed as shown in Figure 2,

The defined rules only transform the structure of $P$ into $P^{\prime}$. The functionalities implemented in $P$ are preserved in $P^{\prime}$. The functions in $P^{\prime}$ are of simpler structures. The predicates that guard the execution of those functions are explicitly defined. Compared to $P, P^{\prime}$ is well structured and easier to test.

Proposition 1. Given an Erlang program $P$ and its $B T P^{\prime}, P^{\prime}$ is functionally equivalent to $P$.

Proof. Given an Erlang program $P$ and its BT transformation $P^{\prime}$, it is sufficient to prove $P^{\prime}$ is functionally equivalent to $P$, if for $f_{i} \in P, i=1, \ldots, m$, the following conditions hold: (1) each unit $c p_{l} \in f_{i}$ has a unique called function $f_{i(l)}^{\prime}$ in $P^{\prime} ;(2)$ the functional behavior (input/output) of $f_{i(l)}^{\prime}$ is identical to 
that of $c p_{l}$ and (3) the called order of the FEB of $f_{i}^{\prime}$ is defined by the order of the executions of $c p_{i}$, namely, $\left(c p_{i(m)} \mid \operatorname{cond}(i(m))=\right.$ true $<<c p_{i(n)} \mid \operatorname{cond}(i(n))=$ true $) \rightarrow$ $\left(f_{i(m)}^{\prime}(\right.$ true, Args $)<<f_{i(m+1)}^{\prime}($ false, Args $)<<\ldots<<f_{i(n-1)}^{\prime}($ false, Args $)<<$ $f_{i(n)}^{\prime}($ true, Args $\left.)\right), \forall(n>m)$.

The proof for the first condition is obvious as the transformation rules define that the relation between a unit and its called counterpart is a one-to-one mapping, that is, for each unit $c p_{i(l)} \in f_{i}$, there is only one called replacement $f_{i(l)}^{\prime}$ in $P^{\prime}$.

The transformation rules define that (1) the functional executions defined in $c p_{i(l)}$ are performed in the FEB of $f_{i(l)}^{\prime} ;(2)$ if $c p_{i(g)} \in f_{i}, g<i$, returns a value to the variable $R V_{i(g)}, R V_{i(g)}$ is defined as an input of $f_{i(l)}^{\prime}$, and (3) the name of $R V_{i(g)}$ is unique (module name plus function name plus variable name). These rules guarantee that the function $f_{i(l)}^{\prime}$ and the unit $c p_{i(l)}$ receive the same input set and perform the same functional executions. Thus, $f_{i(l)}^{\prime}$ and $c p_{i(l)}$ have the same functional behavior.

The third condition can be obtained by contradiction. Let $\left.c p_{i(m)}\right|_{\operatorname{cond}(i(m))=\text { true }}$ $<<\left.p_{i(n)}\right|_{\operatorname{cond}(i(n))=\text { true }}$ be true. This implies that cond $(i(k))=$ false, $m<l<$ $n$, as $c p_{i(m)} \mid \operatorname{cond}(i(m))=$ true $<<c p_{i(n)} \mid \operatorname{cond}(i(n))=$ true $\equiv c p_{i(m)} \mid \operatorname{cond}(i(m))=$ true $<<$ $\left.c p_{i(l)}\right|_{\text {cond }(i(l))=\text { false }}<<\left.c p_{i(n)}\right|_{\text {cond }(i(n))=\text { true }}, \forall(m<k<n)$. Suppose in the transformation counterpart, there exists a called function $f_{i(l)}^{\prime}, m<l<n$, such that $f_{i(l)}^{\prime}($ true, Args) is activated. According to the transformation rules, the PEB of the function $f_{i(l-1)}^{\prime}$ must have invoked the function $f_{i(l)(\operatorname{cond}(i(l)), \text { Args })}^{\prime}$ with cond $(i(l))$ being evaluated to true. This, however, contradicts to the fact that $\operatorname{cond}(i(l))$ is false. Thus, $\left(\left.c p_{i(m)}\right|_{\operatorname{cond}(i(m))=\text { true }}<<\left.c p_{i(n)}\right|_{\operatorname{cond}(i(n))=\text { true }}\right) \rightarrow$ $\left(f_{i(m)}^{\prime}(\right.$ true, Args $)<<f_{i(m+1)}^{\prime}($ false, Args $)<<\ldots<<f_{i(n-1)}^{\prime}($ false, Args $)<<$ $f_{i(n)}^{\prime}($ true, Args $\left.)\right), \forall(n>m)$.

\subsection{Pattern Match Transformation}

Erlang makes extensive use of pattern matching in its function definitions. This work transforms the pattern matching clauses into a binary format. To do so, the technique 4] proposed to eliminate overlapping between patterns are applied. Specifically, pattern match clauses in a function are replaced by a series of called functions, each of which is guarded by the pattern_match function.

A data type called the Structure Splitting Tree (SST) 44 is defined and applied for pattern evaluation, and its use guarantees the pattern match clauses being represented in binary formats.

\subsection{Functional Binary Tree}

After $P$ is transformed into $P^{\prime}$, it is easily to see that all functions in $P^{\prime}$ are represented with a generic pattern as shown in Figure 3 . The pattern is a binary tree with one entry and two exits. The entry is identified with a function name 


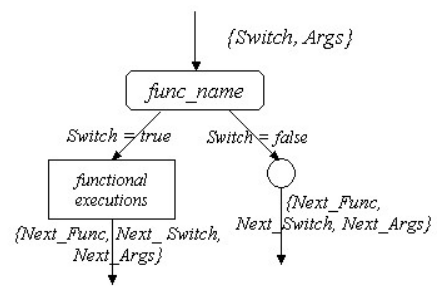

Fig. 3. Generic pattern of the called function

func_name that takes a list of arguments Args as input. Both exits define a tuple $\{$ Next_Func, Next_Switch, Next_Args $\}$ where Next_Func states the function next to be called; Next_Switch defines the guard for Next_Func, and Next_Args consists of the arguments for Next_Func. Switch is applied to guard the selection of the true and the false branches. The true branch defines both the functional executions and the function to be called next, while the false branch only states the next called function. Such a binary tree is defined as a Functional Binary Tree (FBT).

If all called functions in $P^{\prime}$ are represented with their FBTs, a Complete Functional Binary Tree (CFBT) can be derived where the nodes that define no Next_Func constitute the terminals. For example, Figure 4 demonstrates the CFBT of the program shown in Figure 2.

\section{Structural Testing from Transformation}

This section discusses the test generation for structural coverage from the program transformation.

\subsection{Test Generation}

As discussed in the Section 3, a program $P$ can be transformed into its BT $P^{\prime}$. Proposition 1 proves that $P^{\prime}$ is functionally equivalent to $P$. This suggests that, instead of testing $P$ directly, one can test $P^{\prime}$ and use the test results to evaluate the correctness of $P$. Any errors detected in $P^{\prime}$ imply that the implementation of $P$ is faulty. The structure of $P^{\prime}$ is a binary format and the predicates for controlling the execution of a node are explicitly specified. Deriving data to test an execution branch in $P^{\prime}$ should not be a difficult task. Compared to $P, P^{\prime}$ is easier to achieve structural coverage.

This section discusses the derivation of tests for $P^{\prime}$. As $P^{\prime}$ can be represented by a CFBT, it is easy to see that a test set whose elements traverse and test all nodes in the CFBT will test $P^{\prime}$, and the test achieves complete structural coverage. Thus, the generation of a test set for $P^{\prime}$ is achieved by the construction of data that traverse every node in the $C F B T_{P^{\prime}}$ and test the corresponding 


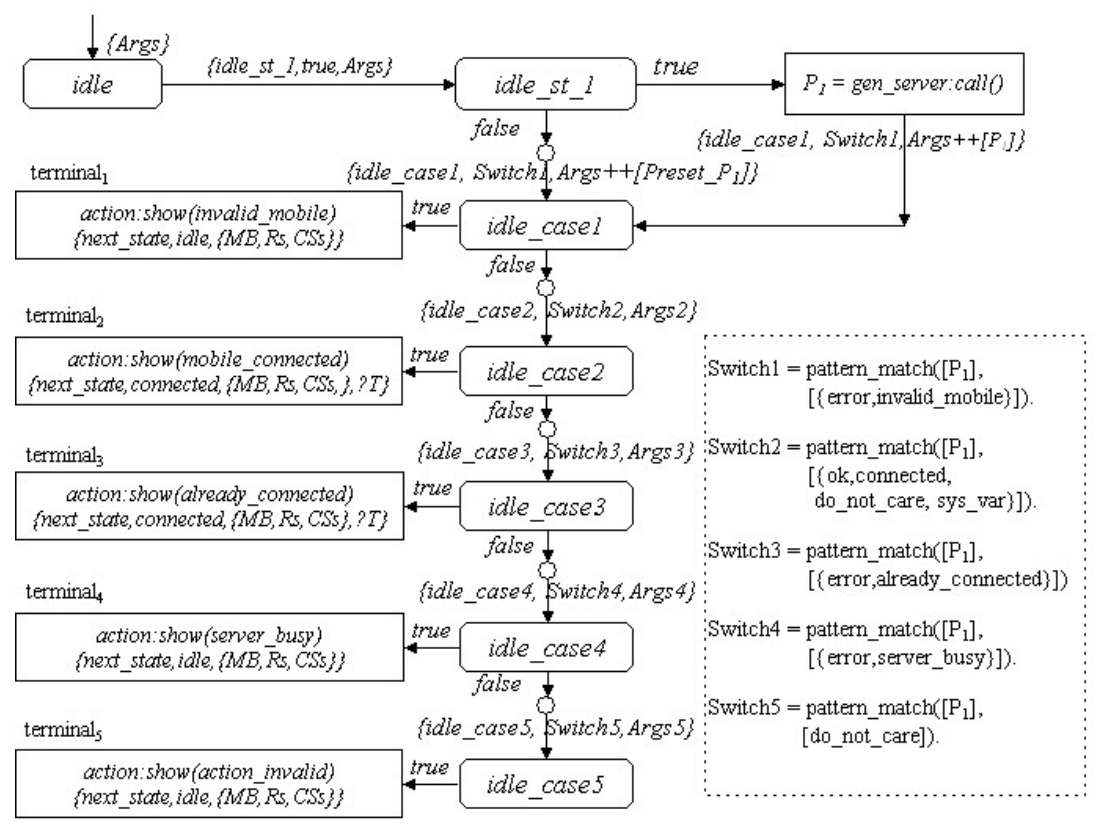

Fig. 4. Complete Functional Binary Tree derived from the program shown in Figure2

function at least once. As a CFBT is a binary tree, standard techniques for the search of nodes in a tree such as a breadth-first search algorithm 3 , or a depth-first search algorithm [3] can be applied.

As an example, a test for the CFBT shown in Figure 4 is defined by constructing a set of data $T S$ whose elements traverse and test each node in the graph at least once. To do so, each terminal terminal te $_{i} \in\left\{\right.$ terminal $_{1}, \ldots$, terminal $\left._{5}\right\}$ needs to be tested by $t s_{i} \subseteq T S$ at least once. Let $t s_{3}$ test terminal ${ }_{3}$. The test $t s_{3}$ is constructed by examining the path between the root node (the entry of the function idle) and the terminal terminal $l_{3}$. The path between terminal $_{3}$ and $i d l e$

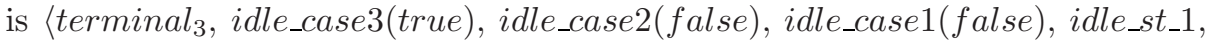
$i d l e\rangle$. The function $i d l e_{-} s t \_1$ can pass $P_{1}$ to its successor. The value of $P_{1}$ can be either computed from the execution gen_server : call() or preset. The subsection 4.2 discusses the technique on deriving partial testing by presetting the inputs for the functions under evaluation.

Thus, in the test, $P_{1}$ should receive $\{$ error, already_connected $\}$ (either received from the gen_server:call() or preset). When the test is applied, the IUT must produce the message already_connected.

Proposition 2. Given a program $P$ and its binary transformed $P^{\prime}$ represented by the complete functional binary tree $C F B T_{P^{\prime}}$, if a test set $T S$ achieves complete structural coverage of $P^{\prime}$, the elements in $T S$ must traverse all nodes in $C F B T_{P^{\prime}}$ and test the corresponding functions at least once and vice versa. 
Proof. Let $n$ be the number of functions in $P^{\prime}$ and $N_{i}$ be the node that stands for the function $f_{i}^{\prime} \in P^{\prime}$ in the $C F B T_{P^{\prime}}$. Suppose a test set $T S$ has achieved complete structural coverage of $P^{\prime}$, there must exist a subset $t s_{i} \subseteq T S$ such that $t s_{i}$ tests the function $f_{i}^{\prime} \in P^{\prime}$, which implies that the function $f_{i}^{\prime}$ is executed by $t s_{i}$ at least once. This is equivalent to that $t s_{i}$ traverses the node $N_{i}$ in the $C F B T_{P^{\prime}}$ at least once. Similarly, if a set of data $t s_{i}$ traverses the node $N_{i}$ in $C F B T_{P^{\prime}}$, it should execute and test the function defined in $N_{i}$ once. The union of all such sets of data constitutes a test set $T S, T S=t s_{1} \cup \ldots \cup t s_{n}$, that achieves complete structural coverage for $P^{\prime}$.

\subsection{Improving Test Controllability and Observability}

Once faults are detected, tests need to be constructed to isolate these faults. The process of identifying the locations of faults is called debugging. The debugging process defines a number of tests, each of which executes a set of designated functions whose outputs should exhibit the properties defined by the design. A test in the debugging process is called a partial test. By observing the results of all partial tests, the faults are expected to be isolated.

The effectiveness of the debugging process is primitively determined by two factors - the test controllability and the test observability. The test controllability determines whether it is always possible to derive a partial test, while the test observability determines whether it is always possible to observe the outputs when the test is executed.

This work shows that, by applying the program transformation, the test controllability and the observability are improved. As discussed in the section 3, in the transformation program $P^{\prime}$, all functions are represented by the BFTs. If $f_{i}^{\prime} \in P^{\prime}$ is further modified into the format as shown in Figure 5 , the function under test is then assigned with two running modes, the normal mode and the debugging mode. In the debugging mode, a debugging framework is associated. The debugging framework is used to track the values of the variables under evaluation, or bypass the functional execution of $f_{i}^{\prime}$ by presetting its switch to false.

For example, in Figure 5, if $f_{i}^{\prime}$ is previously set to the debugging mode, the results of all internal computations $R S_{1}, \ldots, R S_{i}$ will be posted to the debugging framework for comparison. Before $f_{i+1}^{\prime}$ is called, the values of the inputs Switch, $A_{r g}, \ldots, A g_{k}$ will be sent to the debugging framework. The debugging framework returns a tuple $\left\{\right.$ NextMode, DSwitch, $\left.\left[D A r g_{1}, \ldots, D A r g_{k}\right]\right\}$ where NextMode specifies the running mode of $\left.f_{i+1}^{\prime} ; D A r g_{1}, \ldots, D A r g_{k}\right]$ consists of the inputs for $f_{i+1}^{\prime}$ with $D A r g_{l}, 1 \leq l \leq k$, being preset; DSwith saves the value of switch. If DSwith is set to false, the evaluation of $f_{i+1}^{\prime}$ is bypassed and the testing moves on to $f_{i+2}^{\prime}$.

Thus, the configuration of the debugging process can be modelled as shown in Figure 6. Each function $f_{i}^{\prime}$ either takes input values computed from $f_{i-1}^{\prime}$ or preset by the debugging framework. The functional executions of $f_{i}^{\prime}$ can be controlled by presetting the value for switch. If switch is preset to false, the functional 


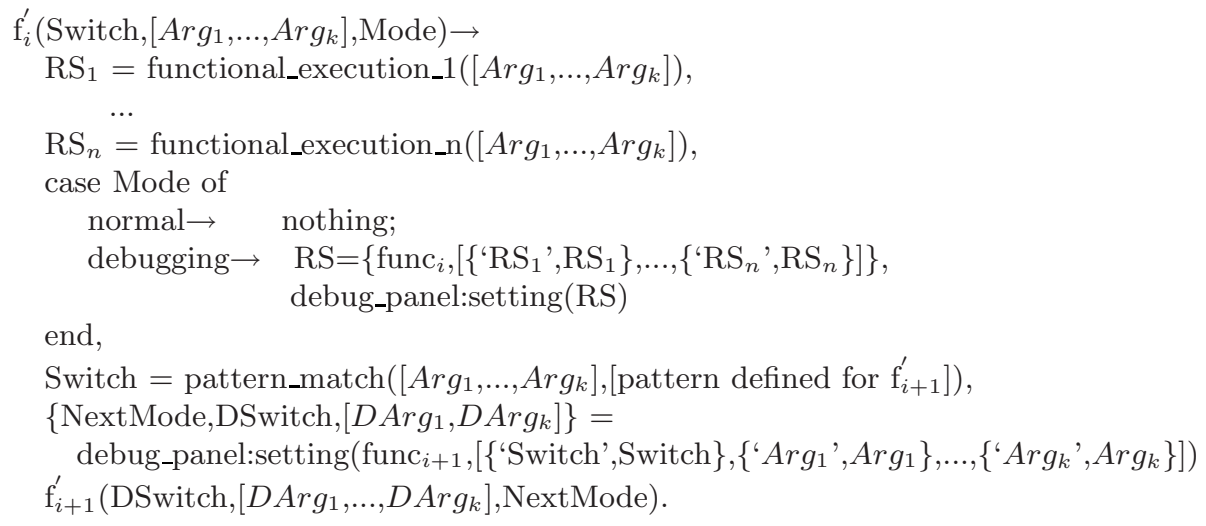

Fig. 5. The generic debugging pattern of a function

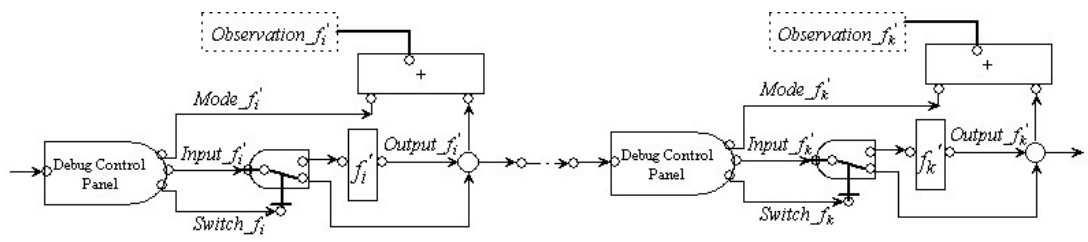

Fig. 6. Debugging framework

execution of $f_{i}^{\prime}$ is bypassed; otherwise, $f_{i}^{\prime}$ will be functionally evaluated. When $f_{i}^{\prime}$ is running on the debugging mode, all computational results performed within the function can be externally observed.

By using the debugging framework to control the values of the inputs for a function under test, the test controllability is improved and, by opening the observation windows in the debugging framework, tracking the changes of the variables under evaluation is possible, which helps to improve the test observability.

\section{A Case Study}

A case study is used to evaluate the proposed model. The case study simulates a telecommunication system.

\subsection{System Infrastructure}

The telecoms uses a client-server structure, and comprises of a database server (DBS) that maintains all client's data and a number of functional servers to process clients' requests. An FS has a capacity that defines the maximum number of mobiles to be connected. 


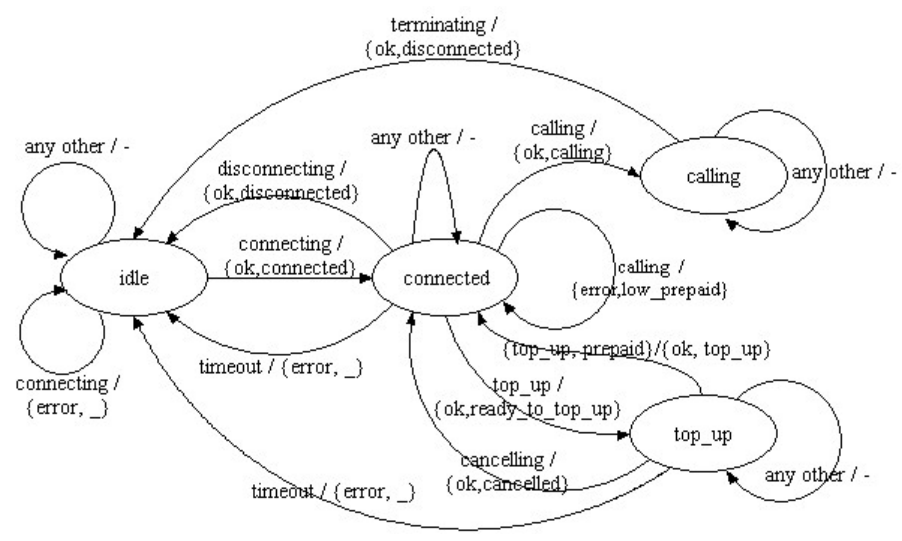

Fig. 7. Client behaviour modelled as an FSM

A client can communicate with any FSs and perform some functional operations such as calling and top-up. Each client has an account maintained in the DBS, and in order to make a phone call, a client needs to top-up enough money in its account. Before performing any functional operations, a client needs to connect to an FS. A client can only be connected to one FS, and if a client has connected to an FS and tries to connect to another FS, the request will be denied.

The behavior of a client (mobile) is modelled as a finite state machine (FSM), and the initial design is shown in Figure 7. There are four states: idle, connected, calling and top_up, where initially, the system is set to the idle state.

The FSM defines the behavior of a number of operations: connecting, disconnecting, calling, terminating, top_up and cancelling. Before performing any operations, a client FSM needs to connect to an FS through sending the connecting request.

A client FSM has a timing restriction applicable when in states connected or top_up. Specifically, when the FSM is directed to the state connected or top_up, a timer will be instantiated which enables the timing process. If, within the predefined time period, no action is performed by the client, a timeout event will be generated and sent to the FS. By receiving timeout event, the FS cuts off the connection and releases the resource from its user list. The FSM is then reset to the state idle.

\subsection{Erlang Implementation}

Erlang is used to implement the telecoms system, making use of the OTP design patterns as is common practice. The FS is implemented using the Erlang/OTP gen_server module. A generic server is implemented by providing a callback 
module where (callback) functions are defined specifying the concrete actions of the server such as server state handling and response to messages.

The client behavior is realized using the OTP gen_fsm module. In accordance with the design, four state functions are defined: idle, connected, calling and top_up. The state function idle shown in Figure 1 initiates a connecting request to an FS.

The state function connected evaluates the requests for the consequent actions. For example, if calling is requested, the function will call the FS to check if the client has saved enough money to make a call. The reply $\{o k$,calling $\}$ enables the client's calling request, which moves the FSM to the state calling.

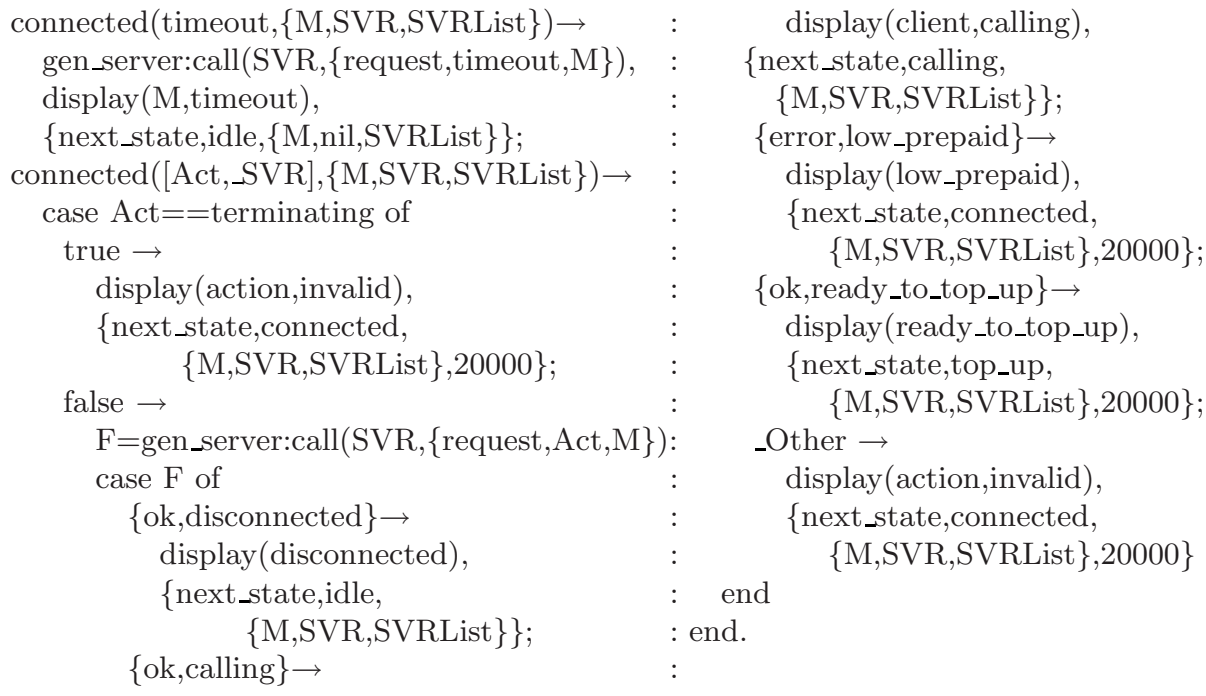

The state function calling enables the calling process and when the FSM is in the state calling, only the terminating action can stop the process. This prevents the calling from being disrupted by any unintended actions.

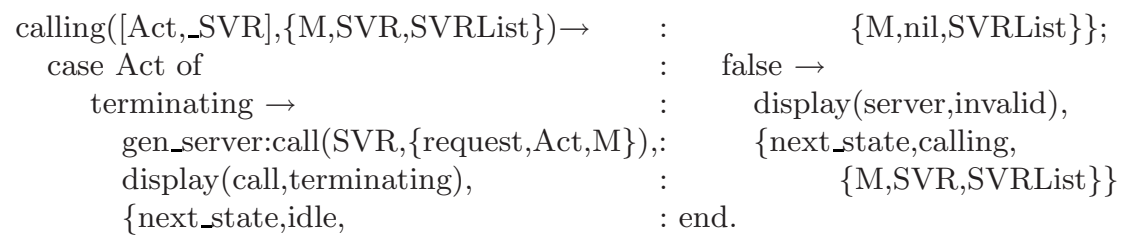

When being in the state connected, the client can ask to top up its account by sending the top_up request to the FS. If $\{o k$,ready_to_top_up $\}$ is replied, the top up process is enabled, and the FSM moves to the state top_up. An action will trigger the state function top_up to either start the transaction by $\left\{t_{0} p_{-} u p\right.$, Prepaid $\}$ operation (Prepaid is the amount of money the client is about to transfer), or cancel the process by sending the cancelling request. 


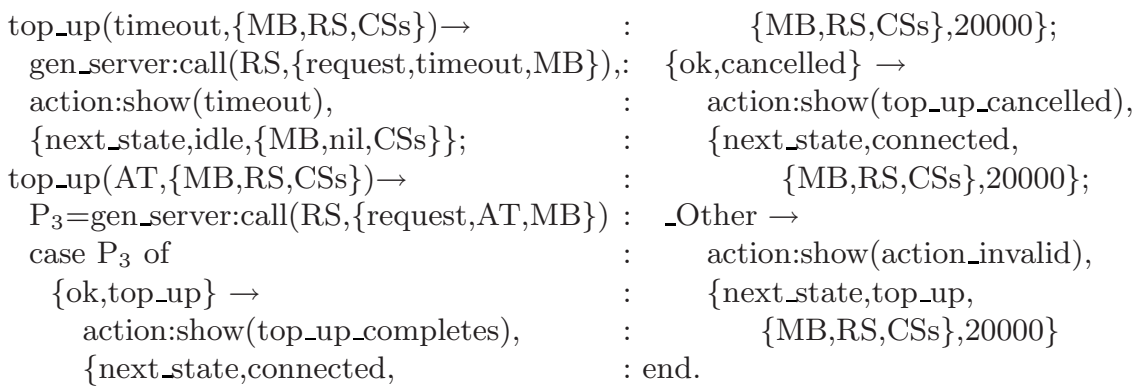

When the FSM moves to the state connected and top_up, a timer is initiated. The timer is set to $20,000 \mathrm{~ms}$. If within the time period, no action is performed, a timeout event will be generated and sent to the FS. The FSM is reset to the state idle. A function command is defined to simulate the receiving of external actions. It calls gen_server:send_event to triggers the state functions.

\subsection{Test Design}

The implementation is tested by applying the proposed testing scheme. The programs of the FS and the client are transformed into the BT formats. The corresponding CFBTs are constructed for test generation. The BT programs are further modified into the debugging mode as discussed in the subsection 4.2 For example, the function idle_st_1 shown in Figure 2 is modified to the debugging mode:

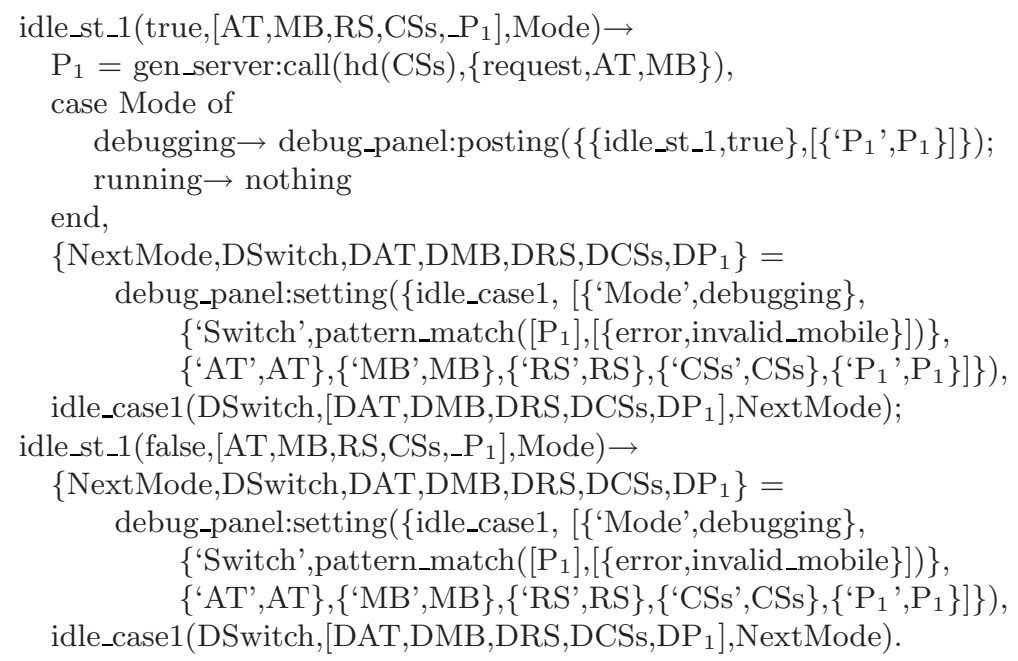

To derive a partial test to check a particular system property, one needs to identify the set of designated functions from the CFBT and preset the inputs for each function by using the debugging framework. For example, to test "A client is the state idle and sends the connecting request to the FS svr 1 . When $\{$ ok, connected,_CalledFS,svr_1\} is replied, the connection is set up". The set of functions is identified from the CFBT (Figure 4) as [idle_st_1, idle_case1, idle_case2]. The settings for the the corresponding functions are: $\left[\{\right.$ idle_st_1, []$\},\left\{\right.$ idle_case1, $\left[\{\right.$ 'Switch',false $\},\left\{\right.$ idle_case $2,\left[\left\{{ }^{\prime} P_{1}\right.\right.$ ', $\{$ ok, 
connected,_CalledFS, svr_1 1$\}]\}]\}]$. The debugging framework presets $P_{1}$ to $\{$ ok, connected, _CalledFS, svr_1 1 and bypasses the functional executions of idle_case1. For each function, the default running mode is debugging. The function idle_st_1 will post the result of $P_{1}$ to the debugging framework for comparison before the executions are completed.

After the inputs being applied, the program should produce the message mobile connected; otherwise, the implementation on such a property is faulty.

\section{Conclusions and Future Work}

Erlang is becoming an increasingly popular language, because it provides a sophisticated platform for the rapid development of concurrent and distributed applications. These often play a business-critical role, which makes testing a crucial component of the Erlang development process. So far, the majority of testing techniques and tools that have been developed for Erlang have focussed on functional testing. Tools such as QuickCheck [2] have become popular because they can rapidely test the system during development.

So far there has been no established technique for the structural testing of Erlang programs. Achieving targets such as branch-coverage has always depended on the ability of a developer to manually identify the necessary sets of test inputs. This paper has presented a technique to automate this process by applying a testability transformation [6]

The basic technique transforms an Erlang IUT into a functionally equivalent counterpart where each atomic function is represented by a binary format called the Functional Binary Tree. These functions are then aggregated into a complete tree (the Complete Functional Binary Tree). An important attribute of the tree is that every predicate that is required to reach any part of the tree is made explicit. The set of tests required to exercise the entire program simply correspond to those tests that are required to explore the entire tree.

A debugging framework is presented to facilitate the observation and manipulation of internal program variables as it is executed. As a function is executed, it provides the ability to track variable values. The execution of specific functions can be bypassed, and the input parameters to functions can be altered via the framework. This is particularly valuable for homing in on faulty areas in the source code.

A small telecoms case study has been presented to illustrate and evaluate the proposed model. By applying the proposed testing scheme, the components in the implementation were transformed into binary formats and then modified into the debugging mode. The CFBT was then constructed to derive the tests.

There still remains much to be done. Future work will apply the techniques here to larger industrial examples. When deriving a partial test to check a particular system property, the proposed model manually checks the CFBT to identify the set of functions. This on occasion can be very time consuming, and it is of interests if one can express the system properties with a formal language such as temporal logic 8], and use the formal expression as a guide to automatically identify the set of functions. This, however, remains a topic for the future work.

\section{Acknowledgements}

This work was funded by the FP7 project ProTest, number 215868: www.protestproject.eu. We're grateful to its academic and industrial members for input to this work and suggestions to improve the process. 


\section{References}

1. Armstrong, J., Virding, R., Wikström, C., Williams, M.: Concurrent Programming in Erlang, 2nd edn. Prentice-Hall, Englewood Cliffs (1996)

2. Arts, T., Hughes, J., Johansson, J., Wiger, U.: Testing Telecoms Software with Quviq Quickcheck. In: Feeley, M., Trinder, P.W. (eds.) Proceedings of the 2006 ACM SIGPLAN Workshop on Erlang (Erlang 2006), pp. 2-10. ACM Press, New York (2006)

3. Bang-Jensen, J., Gutin, G.: Digraphs: Theory Algorithms and Applications. Springer, London (2001)

4. Guo, Q., Derrick, J.: Eliminating overlapping of pattern matching when verifying Erlang programs in $\mu \mathrm{CRL}$. In: 12th International Erlang User Conference (EUC 2006), Stockholm, Sweden (2006)

5. Guo, Q., Derrick, J., Hoch, C.: Verifying Erlang Telecommunication Systems with the Process Algebra $\mu$ CRL. In: Suzuki, K., Higashino, T., Yasumoto, K., El-Fakih, K. (eds.) FORTE 2008. LNCS, vol. 5048, pp. 201-217. Springer, Heidelberg (2008)

6. Harman, M., Hu, L., Hierons, R., Wegener, J., Sthamer, H., Baresel, A.: Testability Transformation. IEEE Transactions on Software Engineering 30(1), 3-16 (2004)

7. King, J.: Symbolic Execution and Program Testing. Communications of the ACM 19(7) (1976)

8. Kröger, F.: Temporal logic of programs. Springer, New York (1987)

9. McMinn, P.: Search-based software test data generation: a survey. Softw. Test, Verif. Reliab. 14(2), 105-156 (2004)

10. Mottu, J.-M., Baudry, B., Traon, Y.: Model Transformation Testing: Oracle Issue. In: Frantzen, L., Merayo, M., Múñez, M. (eds.) 28th International Conference on Software Testing, Verificaiton and Validation Workshop (ICSTW 2008), Washington, DC, USA, pp. 105-112. IEEE Computer Society Press, Los Alamitos (2008)

11. Naslavsky, L., Ziv, H., Richardson, D.J.: Using Model Transformation to Support Model-based Test Coverage Measurement. In: AST 2008: Proceedings of the 3rd international workshop on Automation of software test, pp. 1-6. ACM, New York (2008)

12. Sun, C.-A.: A Transformation-Based Approach to Generating Scenario-Oriented Test Cases from UML Activity Diagrams for Concurrent Applications. In: 32nd Annual IEEE International Computer Software and Applications Conference, pp. 160-167. IEEE Computer Society Press, Los Alamitos (2008)

13. US Department of Transportation, Federal Aviation Administration. Software Approval Guidelines (2003) 8110.49

14. Voas, J., Miller, K.: Software Testability: The New Verification. IEEE Software 12(3), 17-28 (1995)

15. Zhu, H., Hall, P.A.V., May, J.H.R.: Software Unit Test Coverage and Adequacy. ACM Computing Surveys 29(4), 366-427 (1997) 